\title{
Martina Rommel:
}

\section{Sacherschließung Musik für Noten und audiovisuelle Medien in der WLB}

Sie alle kennen die Sacherschließung der WLB für Bücher und andere Materialien: bis 1995 wurde der Systematische Zettelkatalog mit Generalschlagwortregister geführt, seit 1996 wird im Südwestdeutschen Bibliotheksverbund (SWB) nach den RSWK verschlagwortet. Auch Musikbücher, d.h. Abhandlungen über ein bestimmtes Thema in der Musik, z.B. über das Musikleben in Stuttgart oder über die Oper oder eine Mozartbiographie, also die ,Sekundärliteratur', wurden und werden so erschlossen. ,Primärliteratur', wie z.B. Romane oder Gedichte (Schöne Literatur), Kinder- und Jugendbücher, wird dagegen im allgemeinen nur dann sachlich erschlossen, wenn sie ,einen bestimmten, als Problem, Ereignis oder mit einem Namen benennbaren Gegenstand behandelt': z.B. Peter Härtlings Roman über Hölderlin oder ein Kinderbuch über Dinosaurier (RSKW § 4,6). Das ist logisch und einleuchtend: ohne ein solches Thema bietet eine sachliche Erschließung i.d.R. keine zusätzliche Information neben der formalen Erschließung, sie ist damit wenig sinnvoll oder sie wäre - man denke nur an eine Gedichtsammlung - uferlos.

Anders in der Musik als angewandter Kunst. Hier werden Musiknoten und audiovisuelle Medien, die die Primärliteratur in der Musik darstellen, nach verschiedensten Gesichtspunkten gesucht. Die Frage nach dem Werk eines bestimmten Komponisten (oder auch die nach der Einspielung eines bestimmten Interpreten) ist nur eine mögliche Variante. Daneben sucht ein Musiker Noten zum Spielen auf seinem Instrument - z.B. der Geige - oder zum Singen bzw. Tonträger zum Anhören von Kammermusik - z.B. von Streichquartetten - oder von Chormusik egal, wer diese Musik komponiert hat.

Aus diesem Grunde wurde im Musiklesesaal für Musiknoten ein sog. ,Besetzungskatalog' als Zettelkatalog und Teil des Systematischen Kataloges, belegt mit den dort ausgesparten Buchstaben NJ, NK, NL, geführt. Als ,musikalische Besetzung' werden die Musikinstrumente oder Singstimmen bezeichnet, die zur Aufführung einer bestimmten Komposition notwendig sind: beispielsweise ein Streichquartett, ein Sinfonieorchester, ein Duo mit Violine und Klavier, ein Kinderchor etc. ${ }^{1)}$

Die einzelnen Systemstellen des Besetzungskataloges sind wiederum durch ein Schlagwortalphabet der ,musikalischen Formen und Gattungen' das ist z.B. eine Oper oder ein Marsch - untergliedert. Dadurch lassen sich z.B. innerhalb des Orchesters Sinfonien, innerhalb einer Singstimme und Klavier Arien oder Lieder, innerhalb ei- 
ner Klarinette und Orchester Konzerte etc. nachweisen.

Dieser Besetzungskatalog wurde bis Juni 2004 geführt und musste aus zwei Gründen - dem Einstellen des Zetteldrucks und der Digitalisierung des Systematischen Kataloges ${ }^{2)}$ - abgebrochen werden.

Parallel zur Pflege des Besetzungskataloges hat eine ,AG Sacherschließung Musik' des SWB in den letzten anderthalb Jahren Regeln für eine Verschlagwortung von Musiknoten und audiovisuellen Musikmedien nach der musikalischen Besetzung und der musikalischen Form / Gattung im OnlineKatalog erarbeitet. AG-Mitglieder sind die WLB Stuttgart, die PLB Speyer und die Musikhochschulbibliotheken Freiburg, Leipzig, Mannheim, Trossingen. Mit Interesse verfolgt wird das Projekt von der SUB Dresden, der BLB Karlsruhe und der UB Freiburg (teilweise wegen einer eventuellen späteren Mitarbeit), ebenso vom GBV. Durch die Abwanderung der Pfälzischen Landesbibliothek vom SWB zum HBZ finden diese Regeln - die übrigens bei der Tagung der deutschen Gruppe der Internationalen Vereinigung der Musikbibliotheken im September 2005 bundesweit vorgestellt werden sollen - möglicherweise auch dort Anwendung.

Die Regeln sind unabhängig von den RSWK, die Schlagwörter unabhängig von der Schlagwortnormdatei. Für die Schlagwortketten wurden vom Süd- westverbund zehn neue Kategorien (bes, be1 - be9) eingerichtet, für die ein eigener Stichwortindex mit eigenem Suchaspekt (at.bes=) zur Verfügung steht.

Die Trennung von der allgemeinen Sacherschließung ist bewusst und gewollt: sie erlaubt die gezielte Suche z.B. nach Literatur für Violine (Musiknoten oder -tonträger) unabhängig von der Suche nach Literatur über Violine (Sekundärliteratur), bei einer gemeinsamen Verschlagwortung wäre in beiden Fällen jeweils ein Teil des Suchergebnisses überflüssiger Ballast.

Grundlage für die Verschlagwortung sind drei Papiere, die demnächst als SWB-Merkblätter erscheinen werden und auch online auf dem Server des BSZ zur Verfügung stehen werden: 1. Regelwerk (Federführung WLB Stuttgart), 2. Liste „Musikalische Besetzung“ mit Schlagwörtern für Instrumente, Orchester u.ä. (Federführung WLB Stuttgart), 3. Liste „Musikalische Form- und Gattungsnamen“ (Federführung PLB Speyer / MHS Mannheim).

Im Mai wurde mit der Verschlagwortung begonnen, zunächst in einem Probelauf, der im Protokollentwurf der Sitzung der AG Sacherschließung vom 29.9.2004 „als erfolgreich und als strukturell nicht zu verändern bewertet [wurde], so dass die Erfassung ohne Unterbrechung fortgesetzt und in Routine überführt wurde. Zum Stichtag sind knapp 2000 [22.11.2004: 7000] Titel 
mit Ketten zu Besetzung und Gattung erfasst, was durch die beteiligten Bibliotheken und angesichts der Komplexheit des zu beschreibenden Materials als beträchtliche Menge und Erfolg der neuen Erschließung beurteilt wird.“”

Die WLB erschließt auf diese Art und Weise seither den Neuzugang an Musiknoten und erstmalig auch an audiovisuellen Musikmedien nicht nur formal, sondern auch sachlich im SWB.

Da im BISSCAT eine eigene Recherchemöglichkeit für die Musikschlagwörter technisch nicht realisiert werden kann, wird die WLB ihren Benutzern die Recherche nach Musiknoten und audiovisuellen Musikmedien unter dem Aspekt der musikalischen Besetzung und der musikalischen Form / Gattung innerhalb der Pica-Verbunddatenbank (http://swb.bsz-bw.de) anbieten. Diese Möglichkeit besteht, weil (seit dem 16.11.2004) in Pica durch Einschränkung der Suche auf Bibliothekssigel gezielt auf den Bestand der WLB zugegriffen werden kann (Suchanfrage z.B.: „sig 24 AND amk violine“ oder „sig 24 AND amk sinfonie"). Der Musiklesesaal schätzt sich glücklich, dass damit zugunsten der Benutzer ein nahtloser Übergang von der konventionellen Erschließung (Besetzungskatalog) zur Online-Erschließung (über Schlagwort im SWB) realisiert werden konnte.

Eine Einführung und Vorstellung für Mitarbeiter ist vorgesehen, sobald die Pica-Verbunddatenbank, die sich derzeit noch in der Testphase befindet, in den Routinebetrieb überführt ist und das Digitalisierungsprojekt Systematischer Katalog abgeschlossen ist.

1) Dieser Erschließungsaspekt ist für die Primärliteratur der Musik so elementar, dass die Freihandaufstellung in Öffentlichen- und Musikhochschulbibliotheken nach Besetzungskriterien erfolgt.

2) Der Besetzungskatalog liegt inzwischen eingescannt vor. 